\title{
Novel Hetero-Bilayered Materials for Photovoltaics
}

\author{
Jia Zhou ${ }^{1,2, *}$ \\ ${ }^{1}$ Department of Chemistry, Harbin Institute of Technology, Harbin 150001, China \\ ${ }^{2}$ Center for Nanophase Materials Sciences, Oak Ridge National Laboratory, Bethel Valley Road, Oak \\ Ridge, Tennessee 37831-6493 \\ *Corresponding author: E-mail jiazhou@hit.edu.cn
}

\begin{abstract}
The recently synthesized $\mathrm{GaS}$ and $\mathrm{MoSe}_{2}$ nanosheets have been used as appropriate substrates for other layered materials, e.g. silicene/GaS heterosheets akin to graphene/BN systems. Here, we have performed a comprehensive first-principles study of the electronic and optical properties of two-dimensional (2D) GaS/MoSe 2 hetero-bilayers based on density functional theory (DFT). We found almost all proposed GaS/MoSe 2 hetero-bilayers in the current study have an indirect band gap from the $\Gamma$ point to the $\mathrm{K}$ point, except for one with a direct band gap at the $\mathrm{K}$ point. Tunable band gaps $\mathrm{GaS} / \mathrm{MoSe}_{2}$ hetero-bilayers can be controlled by strain modulation. State-of-the-art $G W$-Bethe-Salpeter method, accounting for electron-electron and electron-hole interactions, has been employed to compute accurate absorbance spectra for layered materials. Compared with its composing $\mathrm{GaS}$ and $\mathrm{MoSe}_{2}$ monolayers, GaS/MoSe 2 hetero-bilayers show superior behavior on optical absorbance, indicating a stronger visible-light absorption and applications in solar energy harvesting. We foresee that the novel $\mathrm{GaS} / \mathrm{MoSe}_{2}$ hetero-bilayers would stimulate the fabrication of materials with unprecedented optical and physic-chemical properties that may apply in nanodevices and photovoltaic cells.
\end{abstract}

KEYWORDS: GaS, $\mathrm{MoSe}_{2}$, density functional theory, two dimensional hetero-structures, optical absorbance 


\section{INTRODUCTION}

Graphene, a single atomic layer of graphite, has generated tremendous excitement since its discovery decade ago. ${ }^{1,2}$ It also triggers a boom for other 2D layered materials. 2D layered materials such as graphene, hexagonal BN (h-BN), and transition metal chalcogenides (e.g. GaSe, GaS, MoSe 2 , and $\mathrm{WS}_{2}$ ) remain planar as separated simply by the mechanical ${ }^{3}$ or liquid-phase exfoliation from their bulk layered solids. ${ }^{4}$ The exfoliation is facilitated by the weak interlayer van der Waals (vdW) forces. These 2D layered materials have a broad range of applications, such as field-effect transistors, spin- or valleytronics, thermoelectrics, topological insulators, and energy conversion and storage. ${ }^{5-8}$

(a)
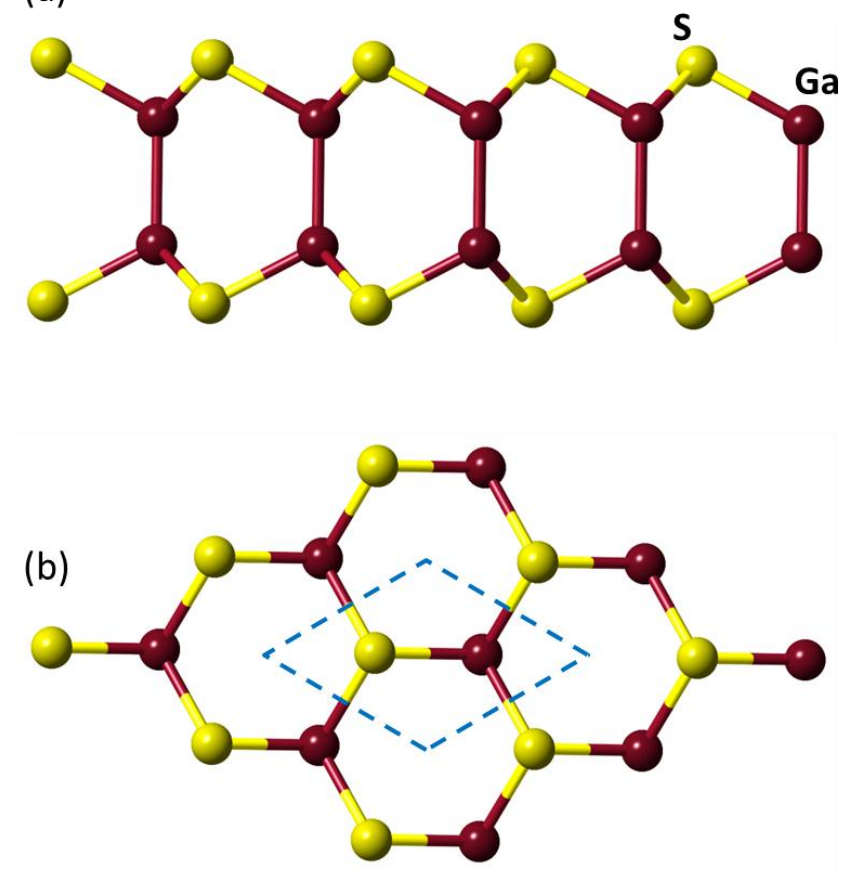

(c)
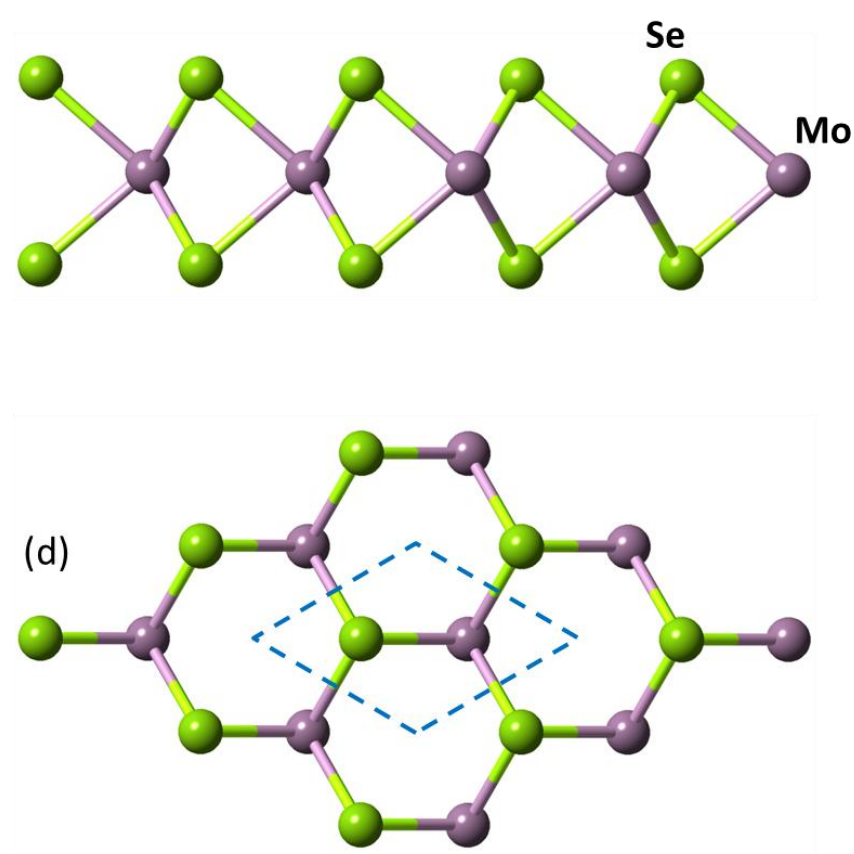

Figure 1. Side view (a) and top view (b) of GaS monolayer with unit cell outlined; Side view (c) and top view (d) of $\mathrm{MoSe}_{2}$ monolayer with unit cell outlined.

As a kind of layered semiconducting material, molybdenum dichalcogenides $\operatorname{MoX}_{2}(\mathrm{X}=\mathrm{S}$, Se) have attracted great interest due to a wide range of important properties. ${ }^{9-11}$ The monolayer of $\mathrm{MoSe}_{2}$ is composed of three sublayers stacked in the sequence $\mathrm{Se}-\mathrm{Mo}-\mathrm{Se}$, as shown in Figure $1 \mathrm{c}\left(\mathrm{MoS}_{2}\right.$ is similar in structure). $\mathrm{MoX}_{2}(\mathrm{X}=\mathrm{S}, \mathrm{Se})$ undergoes a crossover from indirect to direct gap when going 
from bilayer to monolayer, ${ }^{12}$ giving rise to enhanced monolayer luminescence, ${ }^{13}$ thus allowing applications such as transistors, photodetectors and electroluminescent devices. ${ }^{11}$ On the other hand, layered gallium monochalcogenides $\operatorname{GaX}(\mathrm{X}=\mathrm{S}, \mathrm{Se})$ have also drawn much research attention for their potential applications in fields such as solar energy conversion. ${ }^{14}$ Unlike $\mathrm{MoX}_{2}$ monolayer, the monolayer of $\mathrm{GaX}$ consists of four sublayers stacked in the sequence $\mathrm{X}-\mathrm{Ga}-\mathrm{Ga}-\mathrm{X}$, as shown in Figure 1a for GaS monolayer. Experimental efforts have since been carried out to fabricate these $\mathrm{GaX}$ in monolayer form, and monolayer GaS and GaSe sheets have already successfully been synthesized. ${ }^{15-18}$ Soon after, theoretical calculations have also been undertaken to further understand the electronic and photonic properties of GaX monolayers. ${ }^{19,20}$ The GaS monolayer applications in the experiments also include transistors and photodetectors. ${ }^{15-18}$

Recently, the possibility to combine various $2 \mathrm{D}$ materials in vertical stacks creates a new paradigm in materials science: layered hetero-structures. ${ }^{21}$ Those hetero-structures are upheld together by vdW interactions, and thus are called vdW hetero-structures. The hetero-structures have been widely used in conventional semi-conductors for achieving tunable electronic properties. ${ }^{12}$ Graphene/h-BN vdW hetero-structures are one of the first research targets due to their commensurate structural parameters and distinct electronic properties, and a rich collection of physics and functionalities have been revealed by both experimental and theoretical investigations. ${ }^{22-26}$ Moreover, a variety of vdW hetero-structures involving molybdenum dichalcogenides $\operatorname{MoX}_{2}(\mathrm{X}=\mathrm{S}, \mathrm{Se})^{12,27-34}$ and $\mathrm{GaS}^{35}$ have also been studied from both experimental and theoretical perspectives, such as silicene/GaS heterosheets and silicene/MoS 2 heterobilayers. It has been shown that $\mathrm{MoX}_{2}$ and $\mathrm{GaX}(\mathrm{X}=\mathrm{S}, \mathrm{Se})$ are good substrates for other layered materials, such as graphene and silicene, or vise versa. The common ground of these vdW heterostructures is that the in-plane lattice constants of the composing parts are comparable. From our own survey, GaS monolayer and $\mathrm{MoSe}_{2}$ monolayer meet the above criterion. Given the individual excellent behavior of GaS monolayer and $\mathrm{MoSe}_{2}$ monolayer on photoelectrochemistry, we wonder how the novel 
$\mathrm{GaS} / \mathrm{MoSe}_{2}$ hetero-bilayered materials will perform, in particular for photovoltaics, when GaS and $\mathrm{MoSe}_{2}$ monolayers integrate into vdW hetero-bilayers.

In this work, we perform extensive first-principles calculations to characterize novel $\mathrm{GaS} / \mathrm{MoSe}_{2}$ hetero-bilayers, constructed by $\mathrm{GaS}$ and $\mathrm{MoSe}_{2}$ monolayers. Electronic and optical properties of GaS/MoSe 2 hetero-bilayers are systematically investigated by both DFT and $G W$-Bethe-Salpeter method. Our work is expected to pave the way for a new generation of integrated devices and make heterobilayers viable candidates for electronic or photoelectrochemical applications.

\section{COMPUTATIONAL METHODS}

First-principles calculations were carried out using the Vienna ab initio simulation package (VASP). ${ }^{36,37}$ The Kohn-Sham equations were solved using the projector-augmented wave (PAW) method. ${ }^{38,39}$ For the structural relaxations, we employed the Perdew-Burke-Ernzerhof (PBE) functional ${ }^{40,41}$ with the long-range dispersion correction implemented by Grimme. ${ }^{42}$ The Brillouin-zone integrations were performed on a dense $\Gamma$-centered Monkhorst-Pack $16 \times 16 \times 1 \mathrm{k}$-point grid. ${ }^{43}$ The kinetic energy cutoff for plane waves was set to $500 \mathrm{eV}$ and the "accurate" precision setting was adopted to avoid wrap around errors. The convergence criterion for the electronic self-consistent loop was set to $10^{-5} \mathrm{eV}$. During the structural relaxations, the vacuum regions were at least $20 \AA$ to ensure the periodic images are well separated while other lattice vectors were fully relaxed. All atoms were also relaxed until the Hellmann-Feynman forces were smaller than $0.01 \mathrm{eV} / \AA$.

The electron-hole interaction plays an important role in the optical response of a material. ${ }^{44} \mathrm{We}$ calculated the optical spectra of layered materials, including the excitonic interaction, by solving the Bethe-Salpeter equation (BSE), which is implemented in the VASP. ${ }^{45,46}$ The BSE spectrum calculations started with the single-shot $G_{0} W_{0}$ quasiparticle energies and the PBE wave functions. For the more expensive $G_{0} W_{0}$ and $\mathrm{BSE}$ calculations, a $12 \times 12 \times 1 \mathrm{k}$-point grid was applied, which is mostly sufficient for proper description of excitons. ${ }^{47}$ The energy cutoff for the response function was set to $150 \mathrm{eV}$, and the particular pseudopotentials 
provided in VASP for $G W$ calculations (e.g. Mo_sv_GW PAW potential accounting of $4 \mathrm{~s}^{2} 4 \mathrm{p}^{6} 4 \mathrm{~d}^{5} 5 \mathrm{~s}^{1}$ valence electrons for Mo) were used for the $G_{0} W_{0}$ and BSE calculations. A total of 96 bands (more than 4 times of the number of occupied bands in almost all cases ensuring the accuracy of the results) and 96 frequency points were included in the $G_{0} W_{0}$ calculations, which were followed by the BSE calculations. The seven highest valence and seven lowest conduction bands were included in the calculation of the excitonic states for monolayers, while fourteen highest valence and fourteen lowest conduction bands were included for bilayers. Spin-orbit effect at the $G W$ level was within a few tens of meVs, as indicated in the previous study of similar systems, ${ }^{48}$ and was omitted here for sake of computational costs. The adopted computational

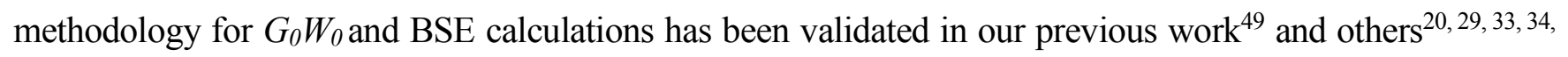
${ }^{48}$ for similar individual monolayer sheets. This approach balances the accuracy and computational costs of our results, and we expect the general trends predicted in this study to be robust.

\section{RESULTS AND DISCUSSION}

For reference, we first study the structures of $\mathrm{GaS}$ and $\mathrm{MoSe}_{2}$ monolayers, as shown in Figure 1. Both of them have a same symmetry as graphene. The calculated lattice constants are $3.58 \AA$ and $3.32 \AA$ for $\mathrm{GaS}$ and $\mathrm{MoSe}_{2}$ monolayers, respectively. These values agree well with previous studies of $\mathrm{GaS}$ and $\mathrm{MoSe}_{2} .{ }^{9}, 20,50$ The closely-matched lattice parameters of $\mathrm{GaS}$ and $\mathrm{MoSe}_{2}$ monolayers make them possible candidates to form hetero-bilayered structure, as seen in Figure 2c, without significantly high strain $\left(\mathrm{GaS}^{19}\right.$ and $\mathrm{MoSe}_{2}{ }^{12}$ monolayers have been studied already with strain up to $10 \%$ and $8 \%$, respectively). For comparison, $\mathrm{GaS} / \mathrm{GaS}$ and $\mathrm{MoSe}_{2} / \mathrm{MoSe}_{2}$ bilayers have also been investigated, which

are shown in Figure $2 \mathbf{a}, \mathbf{b}$. GaS bulk favors the $\beta$ structure, which is AB stacking. ${ }^{51}$ Previous studies of layered transition-metal dichalcogenides (e.g. $\mathrm{MoS}_{2}, \mathrm{MoSe}_{2}$, and $\mathrm{WSe}_{2}$ ) show that only $\mathrm{AB}$ stacking, also called C7 stacking, ${ }^{27}$ gives rise to the lowest energy in most hetero-bilayer systems, and electronic structure is quite insensitive to the stacking. ${ }^{12,27}$ Therefore, for all the bilayers in our current work, the two layers are arranged with AB stacking, so-called C7 stacking. The distances (d in Figure 2) between layers are $3.178 \AA, 3.203 \AA$, and $3.113 \AA$ for fully optimized GaS/GaS bilayer, $\mathrm{MoSe}_{2} / \mathrm{MoSe}_{2}$ bilayer, 
and $\mathrm{GaS} / \mathrm{MoSe}_{2}$ hetero-bilayer, respectively. The closer layer-layer distance in $\mathrm{GaS} / \mathrm{MoSe}_{2}$ heterobilayer than $\mathrm{GaS} / \mathrm{GaS}$ bilayer and $\mathrm{MoSe}_{2} / \mathrm{MoSe}_{2}$ bilayer, as well as the negative interaction energy ($0.17 \mathrm{eV}$ ), indicates at least comparable interaction, if not stronger, between $\mathrm{GaS}$ monolayer and $\mathrm{MoSe}_{2}$ monolayer, and thus could be made in the experiments.

(a)

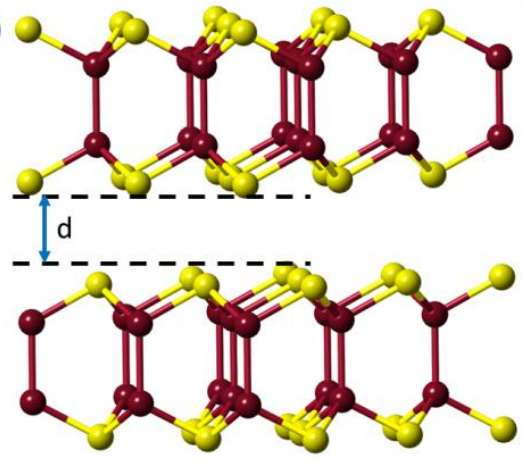

(b)
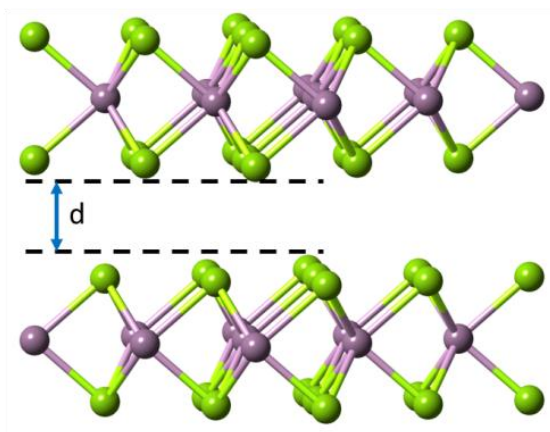

(c)

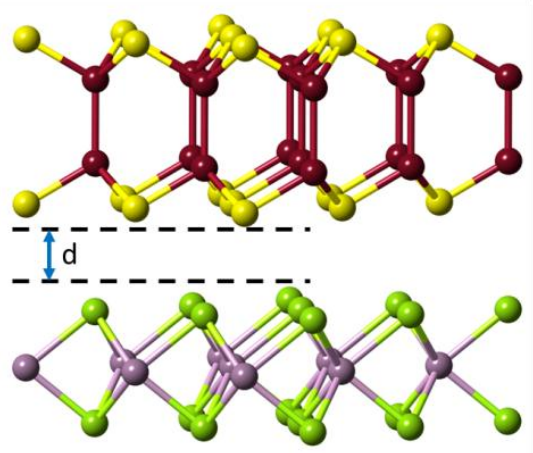

Figure 2. GaS/GaS bilayer (a), $\mathrm{MoSe}_{2} / \mathrm{MoSe}_{2}$ bilayer (b), and $\mathrm{GaS} / \mathrm{MoSe}_{2}$ hetero-bilayer (c). 


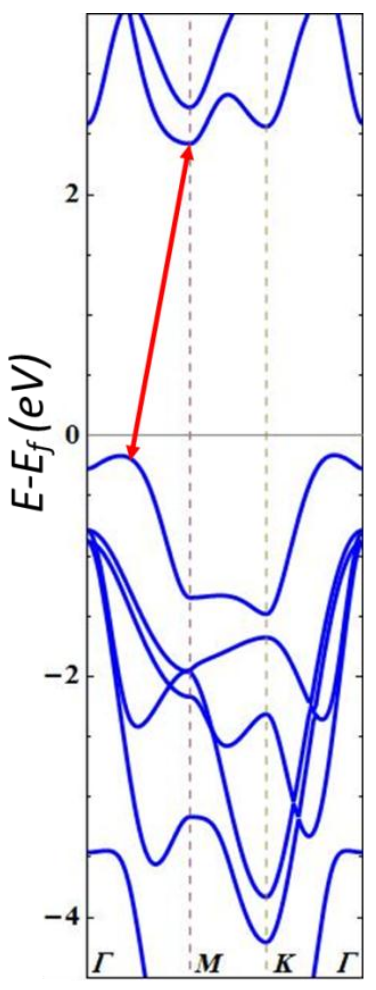

(a)

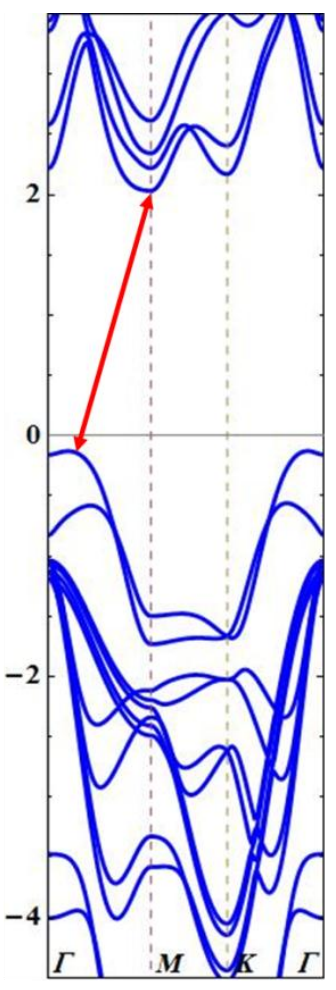

(b)

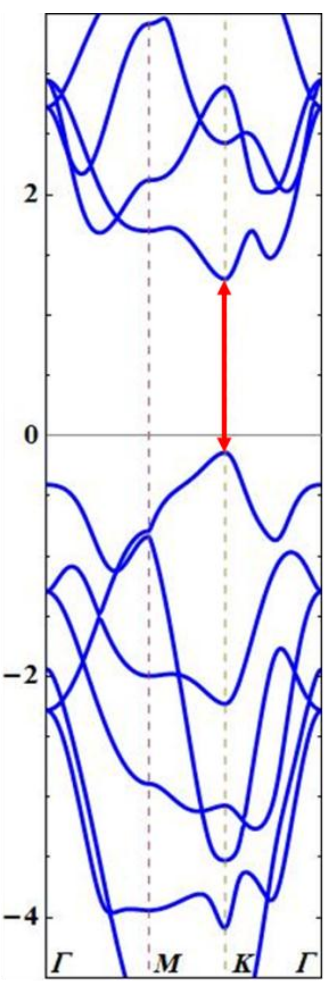

(c)

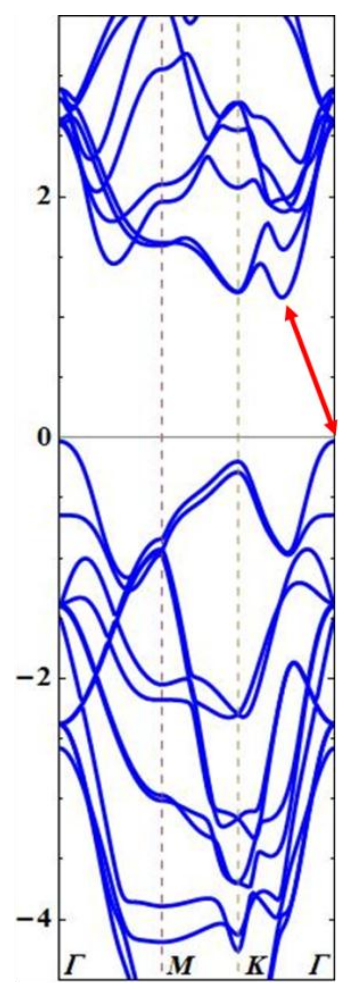

(d)

Figure 3. Band structures of GaS monolayer (a), GaS/GaS bilayer (b), MoSe 2 monolayer (c), and $\mathrm{MoSe}_{2} / \mathrm{MoSe}_{2}$ bilayer (d). The red solid arrows indicate the band gap.

Table 1. Band Gaps ( $E_{\mathrm{g}}$ in $\mathrm{eV}$ ) for layered materials, and positions of a (first peak of the spectrum) and b (the highest peak within the range of visible light in the spectrum) (in eV) in BSE optical spectra

\begin{tabular}{cccc}
\hline & $\mathrm{E}_{\mathrm{g}}\left(\mathrm{PBE} / G_{0} W_{0}\right)$ & $\mathbf{a}$ & $\mathbf{b}$ \\
\hline GaS monolayer & $2.57 / 4.03$ & 3.74 & \\
GaS/GaS bilayer & $2.14 / 3.32$ & 3.76 & \\
$\mathrm{MoSe}_{2}$ monolayer & $1.45 / 2.22$ & 1.69 & 2.36 \\
$\mathrm{MoSe}_{2} / \mathrm{MoSe}_{2}$ bilayer & $1.21 / 1.71$ & 1.73 & 2.72 \\
& & & \\
\hline GaS/MoSe hetero-bilayer & $\mathrm{E}_{\mathrm{g}}\left(\mathrm{PBE} / \mathrm{G}_{0} W_{0}\right)$ & $\mathbf{a}$ & $\mathbf{b}$ \\
with different strains $(\varepsilon)$ & $1.17 / 1.19$ & 1.74 & 2.48 \\
\hline$-4 \%$ & $1.28 / 1.71$ & 1.57 & 2.49 \\
$-3 \%$ & $0.87 / 1.64$ & 1.36 & 2.42 \\
$-1 \%$ & $0.79 / 1.55$ & 1.31 & 2.38 \\
$0 \%$ & $0.53 / 1.26$ & 1.19 & 2.26 \\
$3 \%$ & $0.26 / 0.96$ & 1.05 & 2.15
\end{tabular}


Before we discuss the electronic properties of $\mathrm{GaS} / \mathrm{MoSe}_{2}$ hetero-bilayer, we first study the electronic properties of $\mathrm{GaS}$ and $\mathrm{MoSe}_{2}$ monolayers and their respective homo-bilayers. Figure 3 shows the band structures of $\mathrm{GaS}$ and $\mathrm{MoSe}_{2}$ monolayers, as well as $\mathrm{GaS} / \mathrm{GaS}$ and $\mathrm{MoSe}_{2} / \mathrm{MoSe}_{2}$ bilayers. Only $\mathrm{MoSe}_{2}$ monolayer has a direct band gap at the K point, while all the other three have an indirect band gap. For $\mathrm{MoSe}_{2} / \mathrm{MoSe}_{2}$ bilayer, the valence band maximum (VBM) relocates to the $\Gamma$ point and the conduction band minimum $(\mathrm{CBM})$ moves somewhere between the $\Gamma$ and $\mathrm{K}$ points, compared with $\mathrm{MoSe}_{2}$ monolayer. For GaS monolayer and $\mathrm{GaS} / \mathrm{GaS}$ bilayer, the $\mathrm{CBM}$ is at the $\mathrm{M}$ point, while the VBM is between the $\Gamma$ and $\mathrm{M}$ points. The band gaps are calculated by both PBE and $G_{0} W_{0}$, and are collected in Table 1. These values are in good accord with the previous studies, ${ }^{12,19,20,33,48,50}$ validating the accuracy of our approach. It is well-known that the PBE functional usually underestimates the band gaps ${ }^{52}$ while quasiparticle $G W$ calculation could generally correct them. For GaS monolayer and GaS/GaS bilayer, $G_{0} W_{0}$ band gaps are 1.2 - $1.5 \mathrm{eV}$ over PBE band gaps, while for $\mathrm{MoSe}_{2}$ monolayer and $\mathrm{MoSe}_{2} / \mathrm{MoSe}_{2}$ bilayer, $G_{0} W_{0}$ band gaps are only $0.5-0.8 \mathrm{eV}$ over PBE. It is interesting to note that for GaS monolayer and bilayer, PBE suggests they are both semiconductors with a band gap below $3 \mathrm{eV}$, while $G_{0} W_{0}$ indicates they are insulators with a band gap over $3 \mathrm{eV}$. Since there is no experimental bandgap data for GaS monolayer and bilayer and in some cases $G W$ method overestimates the band gaps, ${ }^{9}$ we only can conclude that the exact band gaps of GaS monolayer and bilayer, should be between PBE and $G_{0} W_{0}$ values. On the other hand, $\mathrm{MoSe}_{2}$ monolayer and bilayer are semiconductors by both $\mathrm{PBE}$ and $G_{0} W_{0}$.

GaS monolayer has a distinct electronic property from $\mathrm{MoSe}_{2}$ monolayer, e.g. much larger band gap (the difference is over $1.5 \mathrm{eV}$ by $G_{0} W_{0}$ ). However, $\mathrm{GaS}$ and $\mathrm{MoSe}_{2}$ monolayers are close in lattice. It would be interesting to investigate the electronic properties of $\mathrm{GaS} / \mathrm{MoSe}_{2}$ hetero-bilayers. At first, we fully relaxed the whole $\mathrm{GaS} / \mathrm{MoSe}_{2}$ hetero-bilayer, and obtained the most stable hetero-bilayered structure with a lattice constant $3.45 \AA$, lying in the middle of those of GaS and MoSe $\mathrm{M}_{2}$ monolayers. Strain modulation has been commonly used in low-dimensional systems to finely tune their electronic 
properties. Figure 4 shows the band structures of various $\mathrm{GaS} / \mathrm{MoSe}_{2}$ hetero-bilayers, with different inplane strains (positive strains indicate tension while negative strains denote compression). Relative to the most stable $\mathrm{GaS} / \mathrm{MoSe}_{2}$ hetero-bilayer, the strain ( $(\varepsilon)$ of various $\mathrm{GaS} / \mathrm{MoSe}_{2}$ hetero-bilayers is from 4\% (the in-plane lattice constant is the same as pristine $\mathrm{MoSe}_{2}$ monolayer) to $3 \%$ (the in-plane lattice constant is the same as pristine GaS monolayer). All the band structures show an indirect band gap, except for that of lattice constant of $\mathrm{MoSe}_{2}$ monolayer in Figure 4a. The band gap of $\mathrm{GaS} / \mathrm{MoSe}_{2}$ hetero-bilayer with $-4 \%$ strain is $1.17 / 1.19 \mathrm{eV}$ by $\mathrm{PBE} / G_{0} W_{0}$ at the $\mathrm{K}$ point, which is significantly decreased compared with that of $\mathrm{MoSe}_{2}$ monolayer $\left(1.45 / 2.22 \mathrm{eV}\right.$ by PBE $\left./ G_{0} W_{0}\right)$. The CBM of other hetero-bilayers remains at the $\mathrm{K}$ point, while the VBM shifts to the $\Gamma$ point. Judging the band structures, it is clear that the highest valence band and the lowest conduction band of hetero-bilayers both come from $\mathrm{MoSe}_{2}$ monolayer, and the VBM shift the $\mathrm{K}$ point to the $\Gamma$ point was also observed in the pristine $\mathrm{MoSe}_{2}$ monolayer with tensile biaxial strains. ${ }^{53}$ The band gaps for all $\mathrm{GaS} / \mathrm{MoSe}_{2}$ hetero-bilayers are collected in Table 1 as well. The band gap reaches the peak at $1.28 / 1.71 \mathrm{eV}$ by PBE/ $G_{0} W_{0}$ in Figure $\mathbf{4 b}$, and then deceases monotonously when the in-plane lattice constant growing.

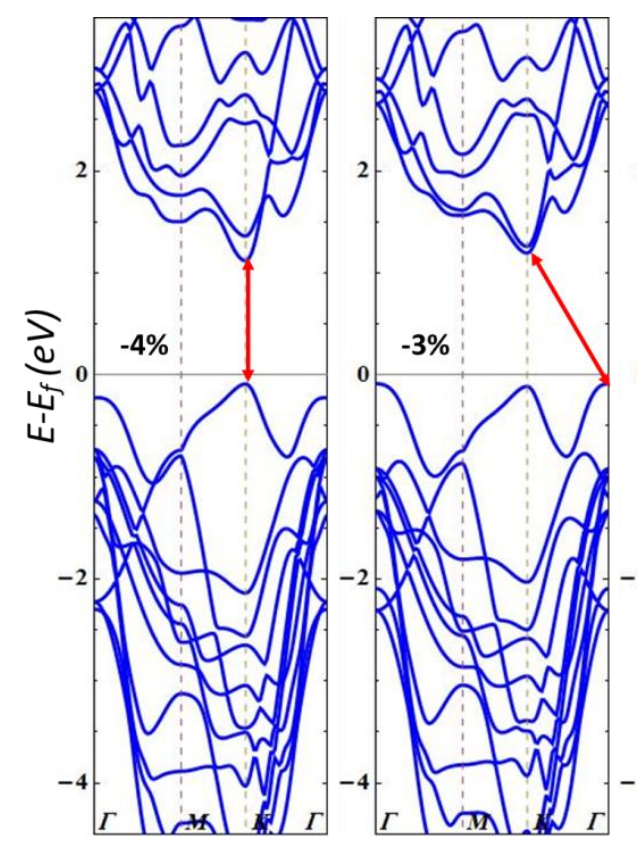

(a) (b)

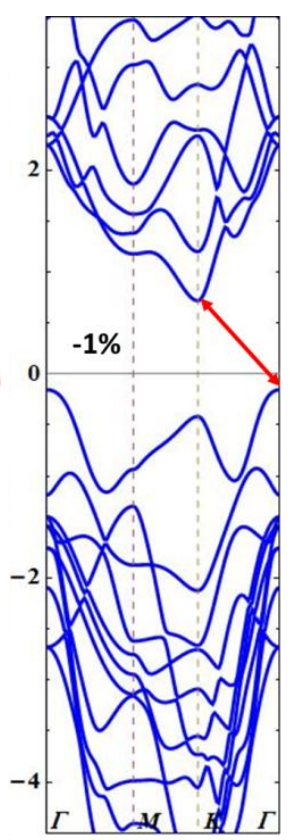

(c)

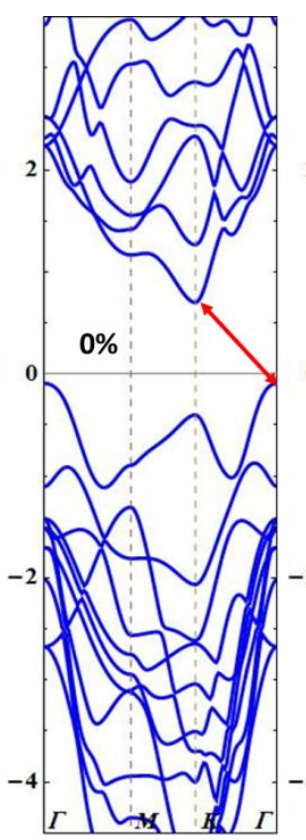

(d)

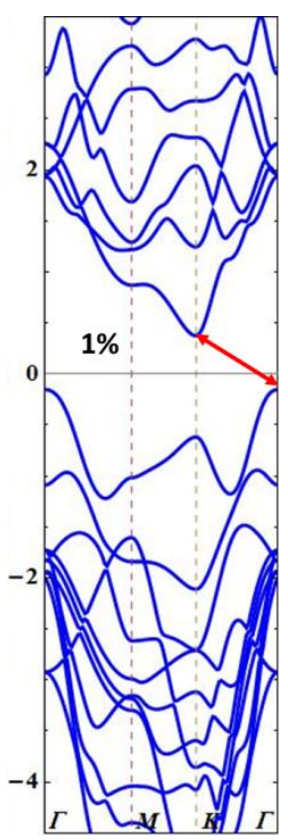

(e)

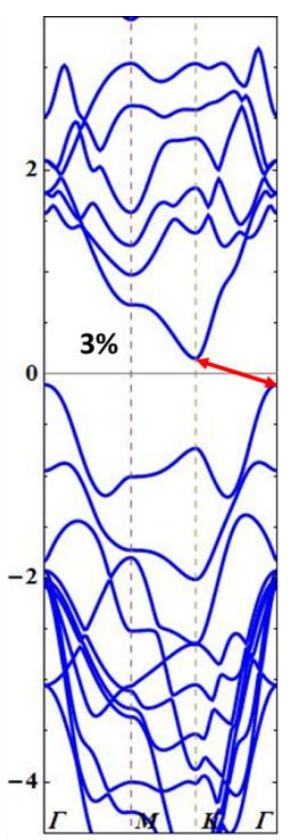

(f)

Figure 4. Band structures of $\mathrm{GaS} / \mathrm{MoSe}_{2}$ hetero-bilayers with different strains: (a) $-4 \%$, (b) $-3 \%$, (c) $1 \%$, (d) $0 \%$, (e) $1 \%$, and (f) $3 \%$. The red solid arrows indicate the band gap. 


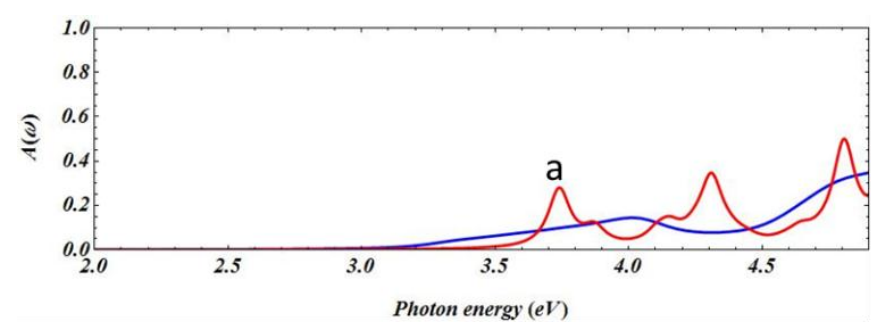

(a)

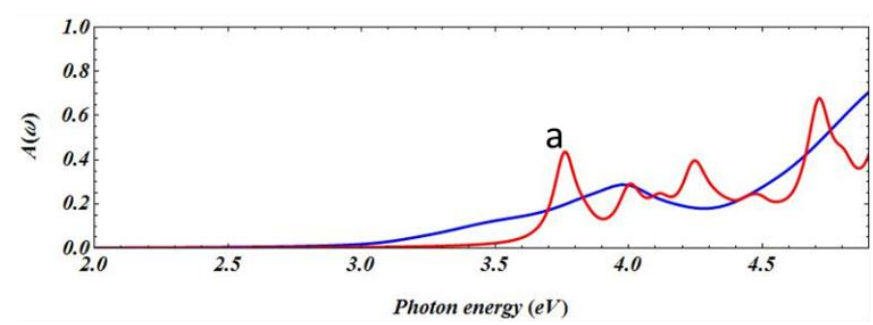

(b)

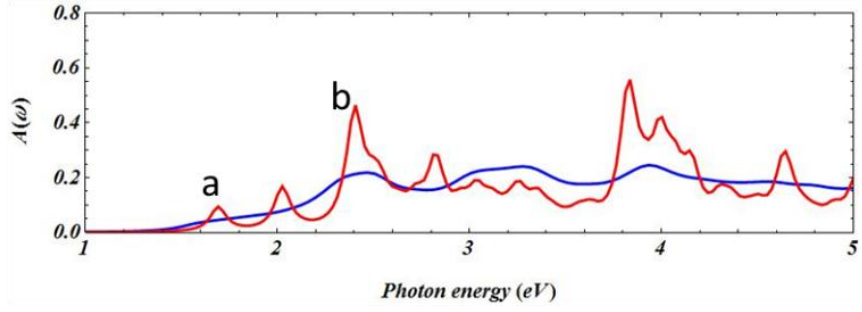

(c)

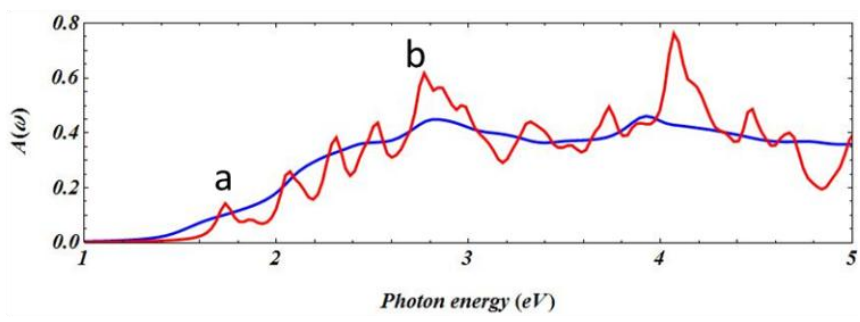

(d)

Figure 5. Optical absorbance spectra $A(\omega)$ (red: GW_BSE; blue: RPA) of GaS monolayer (a), GaS/GaS bilayer (b), $\mathrm{MoSe}_{2}$ monolayer (c), and $\mathrm{MoSe}_{2} / \mathrm{MoSe}_{2}$ bilayer (d). Label a indicates the first peak of $G W \_$BSE curves, while label b indicates the highest peak of $G W \_$BSE curves below $3.26 \mathrm{eV}$.

Next we turn to an important optical property of various GaS/MoSe 2 hetero-bilayers: optical absorbance $A(\omega)$, defined as the fraction of photons of energy $E=\hbar \omega$ absorbed by the 2D sheets. For light with a polarization vector within the plane of the $2 \mathrm{D}$ sheet, $A(\omega)$ is calculated by $A(\omega)=\omega \times L \times \varepsilon_{2} / c$, where $\omega$ is the frequency of photon, $L$ is the interlayer spacing between the isolated layers, $\varepsilon_{2}$ is the imaginary part of the dielectric function, and $c$ is the speed of light in vacuum. ${ }^{54}$ The imaginary part of the dielectric function is calculated by solving the BSE and random-phase approximation (RPA), respectively. Since RPA ignores the electron-hole interaction, its results are not as accurate as those of BSE, and they are shown along with BSE only for the comparison purpose. For comparison, we first study the absorption spectra of $\mathrm{GaS}$ and $\mathrm{MoSe}_{2}$ monolayers, as well as $\mathrm{GaS} / \mathrm{GaS}$ and $\mathrm{MoSe} / \mathrm{MoSe}_{2}$ bilayers, as shown in Figure 5. It is clear that GaS monolayer and bilayer have no absorption below $3.26 \mathrm{eV}$, which is the range of visible light. The first peak of the spectrum (peak a, which is used hereafter) is $3.74 \mathrm{eV}$ and $3.76 \mathrm{eV}$ for GaS monolayer and bilayer, respectively. By contrast, the first peaks of $\mathrm{MoSe}_{2}$ monolayer and bilayer are well below $2.0 \mathrm{eV}$. Our calculated exciton binding energy (the energy difference between the band gap and peak a) of $\mathrm{MoSe}_{2}$ monolayer $(0.53 \mathrm{eV})$ agrees well with the 
previous calculation. ${ }^{33,48}$ Furthermore, the highest absorption of $\mathrm{MoSe}_{2}$ within the range of visible light (1.65 to $3.26 \mathrm{eV}$ ) occurs at $2.36 \mathrm{eV}$ (peak $\mathbf{b}$, which is used hereafter) for monolayer, and $2.72 \mathrm{eV}$ (peak b) for bilayer. The positions of peak $\mathbf{a}$ and $\mathbf{b}$ are collected in Table $\mathbf{1}$. The absorption at the peak $\mathbf{b}$ is over $40 \%$ and $60 \%$ for $\mathrm{MoSe}_{2}$ monolayer and bilayer, respectively.

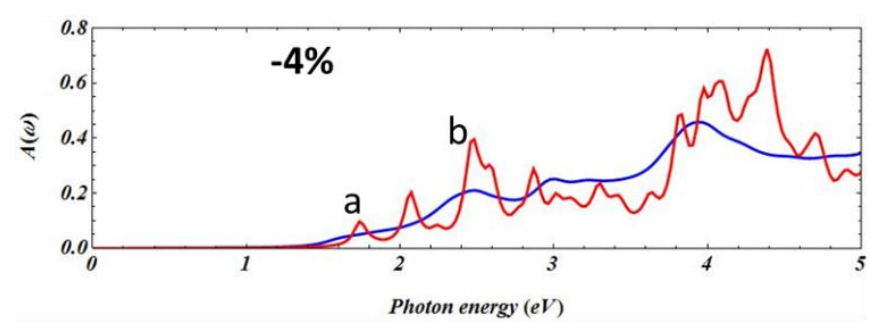

(a)

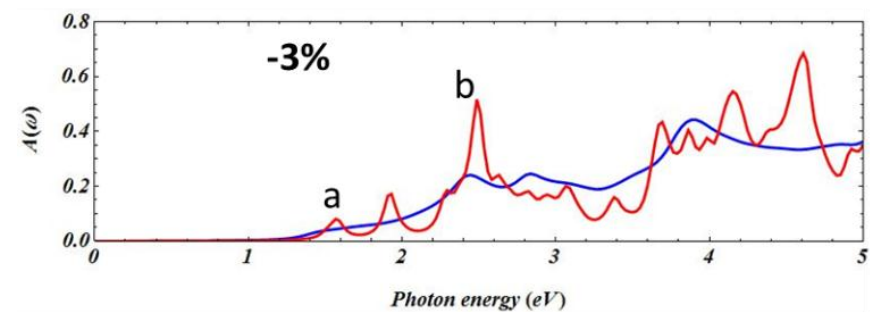

(b)

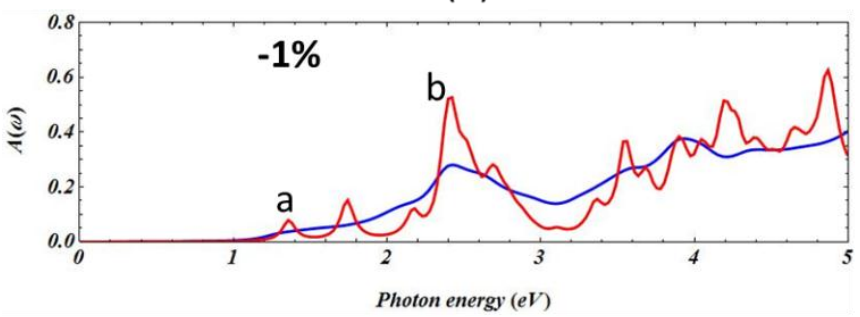

(c)

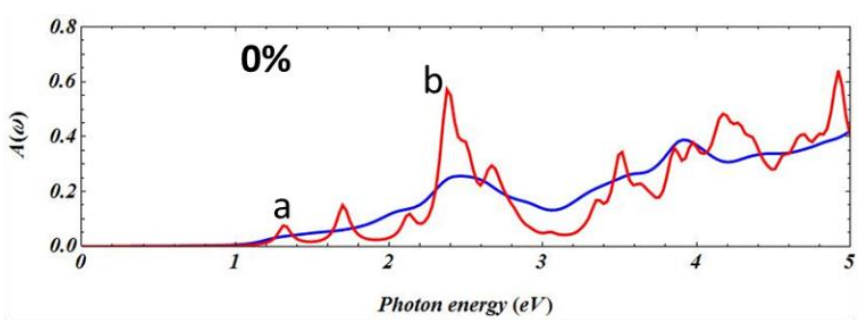

(d)

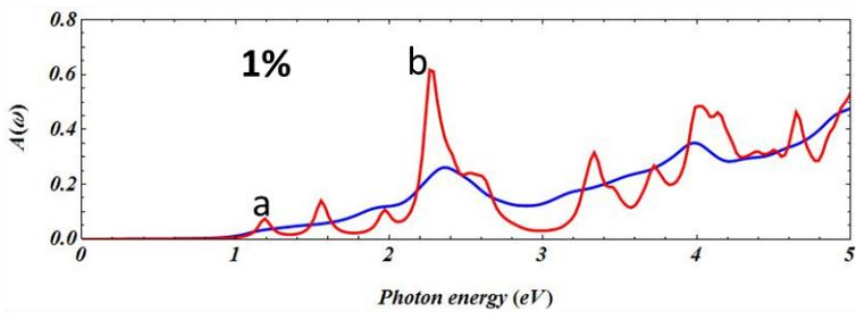

(e)

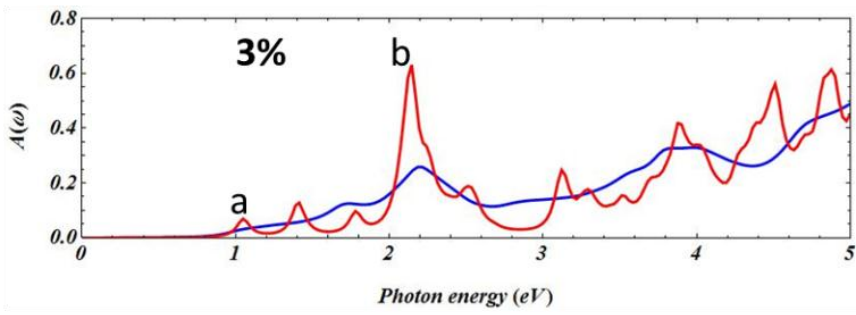

(f)

Figure 6. Optical absorbance spectra $A(\omega)$ (red: $G W \_$BSE; blue: RPA) of GaS/MoSe 2 hetero-bilayers with different strains: (a) $-4 \%$, (b) $-3 \%$, (c) $-1 \%$, (d) $0 \%$, (e) $1 \%$, and (f) $3 \%$. Label a indicates the first peak of $G W \_$BSE curves, while label b indicates the highest peak of $G W \_$BSE curves below $3.26 \mathrm{eV}$.

Figure 6 shows the absorption spectra of various GaS/MoSe 2 hetero-bilayers in accord with Figure 4. The positions of peak $\mathbf{a}$ and $\mathbf{b}$ are collected in Table $\mathbf{1}$ as well. The position of peak $\mathbf{a}$ decreases from $1.74 \mathrm{eV}$ (Figure 6a) to $1.05 \mathrm{eV}$ (Figure 6f) with the increase of the lattice constant. The position of peak b reaches the peak at $2.49 \mathrm{eV}$ (Figure 6b), and goes downhill gradually afterwards. On the other hand, it would be crucial to compare the height of peak $\mathbf{b}$, because it is directly related to the optical absorbance capacity of different 2D layered materials within the range of visible light. From Figure 5, 
GaS monolayer and bilayer have no obvious absorption in the range of visible light, while $A(\omega)$ values of peak $\mathbf{b}$ are 0.47 and 0.61 for $\mathrm{MoSe}_{2}$ monolayer and bilayer, respectively. As for GaS/MoSe 2 heterobilayers, $A(\omega)$ value of peak b increases from 0.40 (Figure 6a) to 0.64 (Figure 6f) with the increase of the in-plane lattice constant. The $A(\omega)$ value of peak $\mathbf{b}$ of the most stable $\mathrm{GaS} / \mathrm{MoSe}_{2}$ hetero-bilayer (Figure 6d) is 0.57 , just slightly lower than that of $\mathrm{MoSe}_{2}$ bilayer. However, peak b in Figure 6d is much sharper than its counterpart in Figure 5d, indicating a much more selective absorption behavior of the light of certain wavelength.

In order to know the detailed contribution from individual layers of $\mathrm{GaS} / \mathrm{MoSe}_{2}$ hetero-bilayer, we study the density of states (DOS) of GaS monolayer, $\mathrm{MoSe}_{2}$ monolayer, and GaS/MoSe 2 hetero-bilayer, as shown in Figure 7a-c. For GaS monolayer, it can be seen that the valence bands from -2 to $0 \mathrm{eV}$ are dominated mainly by S's p state and Ga's p state. On the other hand, the conduction bands from 2 to 4 eV consist mainly of the hybridization from Ga's s and S's p states. For $\mathrm{MoSe}_{2}$ monolayer, however, both the valence bands and the conduction bands within the range from -2 to $4 \mathrm{eV}$ are dominated by Se's p state and Mo's d state. When stacking into GaS/MoSe $\mathrm{H}_{2}$ hetero-bilayer, Mo's d state and Se's $\mathrm{p}$ state from $\mathrm{MoSe}_{2}$ are still dominant in both the valence bands and the conduction bands. However, the components from $\mathrm{GaS}$ are outnumbered significantly by those from $\mathrm{MoSe}_{2}$ near the Fermi level. Also as mentioned previously, GaS monolayer has no absorption below $3.26 \mathrm{eV}$, while $\mathrm{MoSe}_{2}$ monolayer does. That raises the question if the optical absorbance of $\mathrm{GaS} / \mathrm{MoSe}_{2}$ hetero-bilayer mainly comes from the $\mathrm{MoSe}_{2}$ part, only with some structural strain from its free monolayer. Figure 7d shows optical absorbance spectra $A(\omega)$ of most stable $\mathrm{GaS} / \mathrm{MoSe} e_{2}$ hetero-bilayer and of its composing $\mathrm{MoSe}_{2}$ monolayer (directly taken from GaS/MoSe 2 hetero-bilayer), computed by BSE. It is clear that the absorbance spectrum of $\mathrm{GaS} / \mathrm{MoSe}_{2}$ hetero-bilayer is blue shift compared with that of its composing $\mathrm{MoSe}_{2}$ monolayer. Furthermore, the peak $\mathbf{b}$ of $\mathrm{GaS} / \mathrm{MoSe}_{2}$ hetero-bilayer is 0.57 compared with 0.46 (very close to 0.47 of free $\mathrm{MoSe}_{2}$ monolayer itself) of the composing $\mathrm{MoSe}_{2}$ monolayer. It is almost a 
quarter increase of the optical absorbance of $\mathrm{MoSe}_{2}$ monolayer, indicating a significantly positive contribution from GaS monolayer, not by structural strain from free $\mathrm{MoSe}_{2}$ monolayer itself.

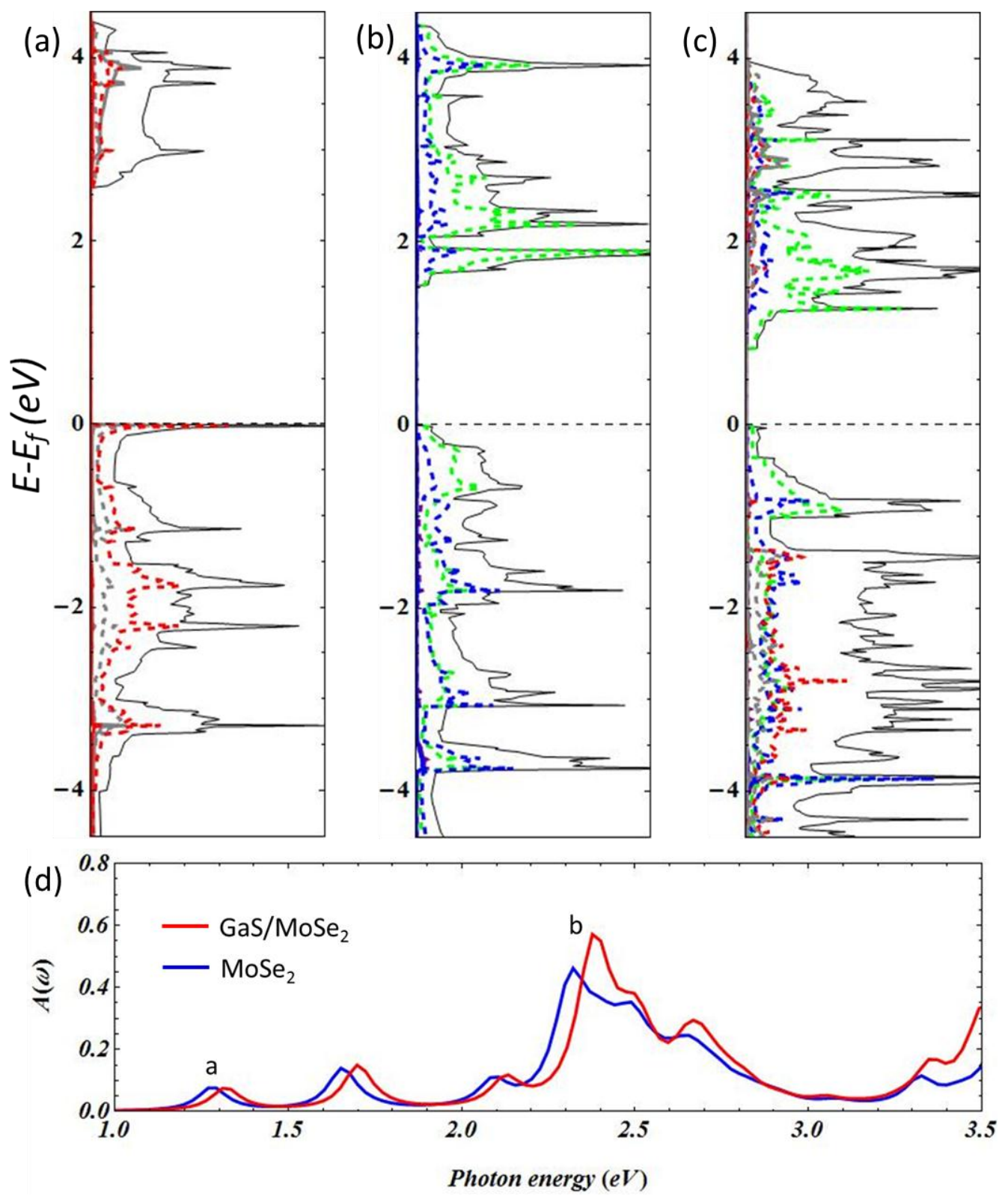

Figure 7. Total/partial DOS of GaS monolayer (a), $\mathrm{MoSe}_{2}$ monolayer (b), and $\mathrm{GaS} / \mathrm{MoSe}_{2}$ heterobilayer (c) (black: total DOS; solid gray: Ga-s pDOS; dashed gray: Ga-p pDOS; solid red: S-s pDOS; dashed red: S-p pDOS; solid purple: Mo-s pDOS; dashed purple: Mo-p pDOS; dashed green: Mo-d pDOS; solid blue: Se-s pDOS; dashed blue: Se-p pDOS). (d) Optical absorbance spectra $A(\omega)$ of the most stable GaS/MoSe 2 hetero-bilayer (red) and of its composing $\mathrm{MoSe}_{2}$ monolayer (blue), computed using BSE. 


\section{CONCLUSION}

In summary, we have investigated the electronic and optical properties of various GaS/MoSe 2 heterobilayers by DFT and state-of-the-art many-body perturbation theory including quasiparticle $G W$ approach and BSE. Even though GaS monolayer has a distinct electronic property from $\mathrm{MoSe}_{2}$ monolayer, they have the same symmetry with close lattice constants, making them good candidates to form $\mathrm{GaS} / \mathrm{MoSe}_{2}$ hetero-bilayers. Most $\mathrm{GaS} / \mathrm{MoSe}_{2}$ hetero-bilayers in our current study have an indirect band gap from the $\Gamma$ point to the K point. With respect to the optical absorbance, GaS/MoSe 2 heterobilayers show superior behavior compared with its composing GaS and $\mathrm{MoSe}_{2}$ monolayers. These theoretical predictions suggest that $\mathrm{GaS} / \mathrm{MoSe}_{2}$ hetero-bilayers might be very promising for optoelectronic applications at the nanometer scale.

\section{ACKNOWLEDGEMENTS}

This research is supported by the Fundamental Research Funds for the Central Universities of China (Grant No. AUGA5710013115). This work used computational resources of the Oak Ridge Leadership Computing Facility at Oak Ridge National laboratory and of the National Energy Research Scientific

Computing Center, which are supported by the Office of Science of the U.S. Department of Energy under Contract No. DE-AC05-00OR22750 and DE-AC02-05CH11231, respectively. We acknowledge the support from the Center for Nanophase Materials Sciences, which is sponsored at ORNL by the Scientific User Facilities Division, U.S. Department of Energy. The author also thanks Dr. Jingsong Huang (ORNL, US) and Prof. Xiaoming Cao (ECUST, China) for the assistance on the computations.

\section{ADDITIONAL INFORMATION}

Competing financial interests: The author declares no competing financial interests. 


\section{References}

1. K. S. Novoselov, A. K. Geim, S. V. Morozov, D. Jiang, Y. Zhang, S. V. Dubonos, I. V. Grigorieva and A. A. Firsov, Science, 2004, 306, 666-669.

2. K. S. Novoselov, A. K. Geim, S. V. Morozov, D. Jiang, M. I. Katsnelson, I. V. Grigorieva, S. V. Dubonos and A. A. Firsov, Nature, 2005, 438, 197-200.

3. K. S. Novoselov, D. Jiang, F. Schedin, T. J. Booth, V. V. Khotkevich, S. V. Morozov and A. K. Geim, Proc. Natl. Acad. Sci. USA, 2005, 102, 10451-10453.

4. J. N. Coleman, M. Lotya, A. O’Neill, S. D. Bergin, P. J. King, U. Khan, K. Young, A. Gaucher, S. De, R. J. Smith, I. V. Shvets, S. K. Arora, G. Stanton, H.-Y. Kim, K. Lee, G. T. Kim, G. S. Duesberg, T. Hallam, J. J. Boland, J. J. Wang, J. F. Donegan, J. C. Grunlan, G. Moriarty, A. Shmeliov, R. J. Nicholls, J. M. Perkins, E. M. Grieveson, K. Theuwissen, D. W. McComb, P. D. Nellist and V. Nicolosi, Science, 2011, 331, 568-571.

5. S. Z. Butler, S. M. Hollen, L. Cao, Y. Cui, J. A. Gupta, H. R. Gutiérrez, T. F. Heinz, S. S. Hong, J. Huang, A. F. Ismach, E. Johnston-Halperin, M. Kuno, V. V. Plashnitsa, R. D. Robinson, R. S. Ruoff, S. Salahuddin, J. Shan, L. Shi, M. G. Spencer, M. Terrones, W. Windl and J. E. Goldberger, ACS Nano, 2013, 7, 2898-2926.

6. C. N. R. Rao, H. S. S. Ramakrishna Matte and U. Maitra, Angew. Chem. Int. Ed., 2013, 52, $13162-13185$.

7. $\quad$ Q. Tang and Z. Zhou, Prog. Mater. Sci., 2013, 58, 1244-1315.

8. $\quad$ P. Miro, M. Audiffred and T. Heine, Chem. Soc. Rev., 2014, 43, 6537-6554.

9. C. Ataca, H. Şahin and S. Ciraci, J. Phys. Chem. C, 2012, 116, 8983-8999.

10. W. S. Yun, S. W. Han, S. C. Hong, I. G. Kim and J. D. Lee, Phys. Rev. B, 2012, 85, 033305.

11. Q. H. Wang, K. Kalantar-Zadeh, A. Kis, J. N. Coleman and M. S. Strano, Nat Nano, 2012, 7, 699-712.

12. N. Lu, H. Guo, L. Li, J. Dai, L. Wang, W.-N. Mei, X. Wu and X. C. Zeng, Nanoscale, 2014, 6, $2879-2886$.

13. S. Tongay, J. Zhou, C. Ataca, K. Lo, T. S. Matthews, J. Li, J. C. Grossman and J. Wu, Nano Lett., 2012, 12, 5576-5580.

14. A. Aruchamy, Photoelectrochemistry and photovoltaics of layered semiconductors, Springer, 1992.

15. P. Hu, Z. Wen, L. Wang, P. Tan and K. Xiao, ACS Nano, 2012, 6, 5988-5994.

16. D. J. Late, B. Liu, J. Luo, A. Yan, H. S. S. R. Matte, M. Grayson, C. N. R. Rao and V. P. Dravid, Adv. Mater., 2012, 24, 3549-3554.

17. D. J. Late, B. Liu, H. S. S. R. Matte, C. N. R. Rao and V. P. Dravid, Adv. Funct. Mater., 2012, 22, 1894-1905.

18. S. Lei, L. Ge, Z. Liu, S. Najmaei, G. Shi, G. You, J. Lou, R. Vajtai and P. M. Ajayan, Nano Lett., 2013, 13, 2777-2781.

19. Y. Ma, Y. Dai, M. Guo, L. Yu and B. Huang, Phys. Chem. Chem. Phys., 2013, 15, 7098-7105.

20. H. L. Zhuang and R. G. Hennig, Chem. Mater., 2013, 25, 3232-3238.

21. L. Britnell, R. M. Ribeiro, A. Eckmann, R. Jalil, B. D. Belle, A. Mishchenko, Y.-J. Kim, R. V. Gorbachev, T. Georgiou, S. V. Morozov, A. N. Grigorenko, A. K. Geim, C. Casiraghi, A. H. C. Neto and K. S. Novoselov, Science, 2013, 340, 1311-1314.

22. C. R. Dean, A. F. Young, I. Meric, C. Lee, L. Wang, S. Sorgenfrei, K. Watanabe, T. Taniguchi, P. Kim, K. L. Shepard and J. Hone, Nature Nanotech., 2010, 5, 722-726.

23. Y. Fan, M. Zhao, Z. Wang, X. Zhang and H. Zhang, Appl. Phys. Lett., 2011, 98, 083103.

24. J. Xue, J. Sanchez-Yamagishi, D. Bulmash, P. Jacquod, A. Deshpande, K. Watanabe, T. Taniguchi, P. Jarillo-Herrero and B. J. LeRoy, Nature Mater., 2011, 10, 282-285. 
25. M. Yankowitz, J. Xue, D. Cormode, J. D. Sanchez-Yamagishi, K. Watanabe, T. Taniguchi, P. Jarillo-Herrero, P. Jacquod and B. J. LeRoy, Nature Phys., 2012, 8, 382-386.

26. W. Yang, G. Chen, Z. Shi, C.-C. Liu, L. Zhang, G. Xie, M. Cheng, D. Wang, R. Yang, D. Shi, K. Watanabe, T. Taniguchi, Y. Yao, Y. Zhang and G. Zhang, Nature Mater., 2013, 12, 792-797.

27. K. Kośmider and J. Fernández-Rossier, Phys. Rev. B, 2013, 87, 075451.

28. H. Terrones, F. López-Urías and M. Terrones, Sci. Rep., 2013, 3, 1549.

29. M. Bernardi, M. Palummo and J. C. Grossman, Nano Lett., 2013, 13, 3664-3670.

30. X. Li, Y. Dai, Y. Ma, Q. Liu and B. Huang, Nanotechnology, 2015, 26, 135703.

31. N. Gao, J. C. Li and Q. Jiang, Phys. Chem. Chem. Phys., 2014, 16, 11673.

32. D. Chiappe, E. Scalise, E. Cinquanta, C. Grazianetti, B. van den Broek, M. Fanciulli, M. Houssa and A. Molle, Adv. Mater., 2014, 26, 2096-2101.

33. M. M. Ugeda, A. J. Bradley, S.-F. Shi, F. H. da Jornada, Y. Zhang, D. Y. Qiu, W. Ruan, S.-K. Mo, Z. Hussain, Z.-X. Shen, F. Wang, S. G. Louie and M. F. Crommie, Nat. Mater., 2014, 13, 1091-1095.

34. H.-P. Komsa and A. V. Krasheninnikov, Phys. Rev. B, 2013, 88, 085318.

35. Y. Ding and Y. Wang, Appl. Phys. Lett., 2013, 103, 043114.

36. G. Kresse and J. Furthmüller, Phys. Rev. B, 1996, 54, 11169-11186.

37. M. Shishkin and G. Kresse, Phys. Rev. B 2006, 74, 035101.

38. P. E. Blöchl, Phys. Rev. B, 1994, 50, 17953-17979.

39. G. Kresse and D. Joubert, Phys. Rev. B, 1999, 59, 1758-1775.

40. J. P. Perdew, K. Burke and M. Ernzerhof, Phys. Rev. Lett., 1996, 77, 3865-3868.

41. J. P. Perdew, K. Burke and M. Ernzerhof, Phys. Rev. Lett., 1997, 78, 1396-1396.

42. S. Grimme, J. Comput. Chem., 2006, 27, 1787-1799.

43. H. J. Monkhorst and J. D. Pack, Phys. Rev. B, 1976, 13, 5188-5192.

44. G. Onida, L. Reining and A. Rubio, Rev. Mod. Phys. , 2002, 74, 601-659.

45. P. Rinke, A. Schleife, E. Kioupakis, A. Janotti, C. Rödl, F. Bechstedt, M. Scheffler and C. G. Van de Walle, Phys. Rev. Lett., 2012, 108, 126404.

46. C. Rödl and F. Bechstedt, Phys. Rev. B, 2012, 86, 235122.

47. A. Molina-Sánchez, D. Sangalli, K. Hummer, A. Marini and L. Wirtz, Phys. Rev. B 2013, 88, 045412.

48. H.-P. Komsa and A. V. Krasheninnikov, Phys. Rev. B, 2012, 86, 241201.

49. J. Zhou, B. G. Sumpter, P. R. C. Kent and J. Huang, ACS Appl. Mater. Interfaces, 2015, 7, 14581464.

50. W. Huang, H. Da and G. Liang, J. Appl. Phys., 2013, 113, 104304

51. Z. Zhu, Y. Cheng and U. Schwingenschlögl, Phys. Rev. Lett., 2012, 108, 266805.

52. J. P. Perdew, Int. J. Quantum Chem., 1985, 28, 497-523.

53. C.-H. Chang, X. Fan, S.-H. Lin and J.-L. Kuo, Phys. Rev. B, 2013, 88, 195420.

54. L. Yang, J. Deslippe, C.-H. Park, M. L. Cohen and S. G. Louie, Phys. Rev. Lett. , 2009, 103, 186802. 


\section{TOC}

$\mathrm{GaS} / \mathrm{MoSe}_{2}$ hetero-bilayers show superior behavior on optical absorbance, indicating a strong visiblelight absorption and applications in solar energy harvesting
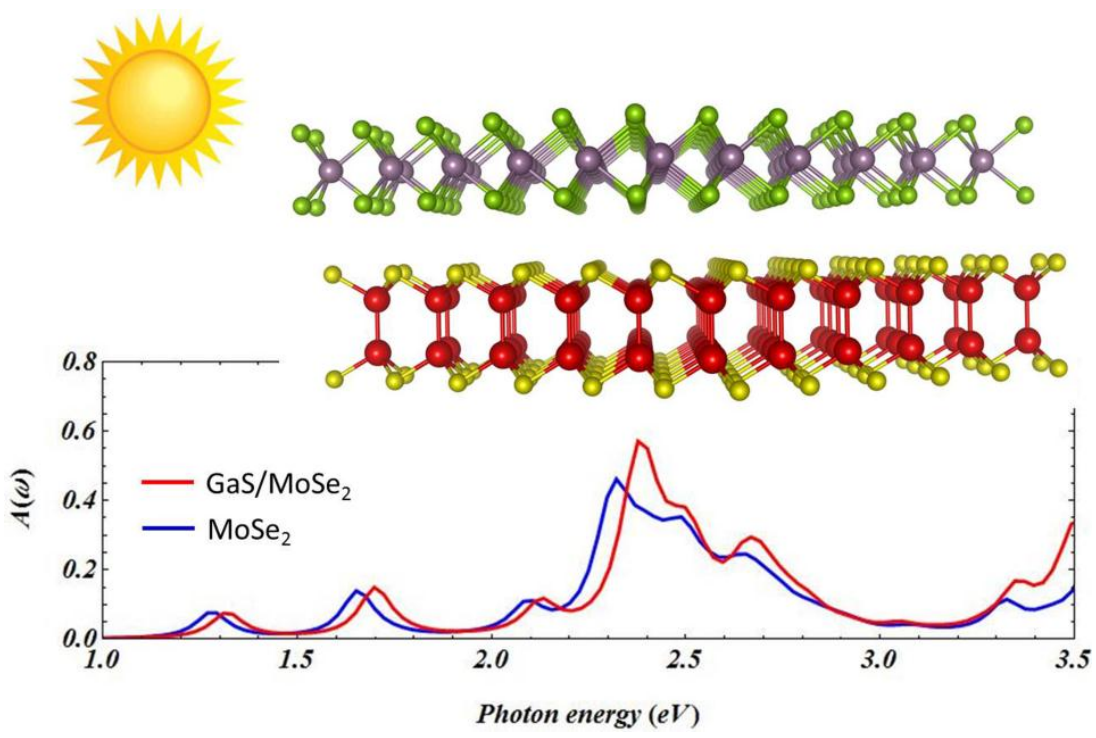